\title{
Vergleichende Methoden zur quantitativ immunologischen Bestimmung und Ermittlung von Normbereichen der Immunglobuline G, A, M, des Haptoglobins und Transferrins mit Hilfe der automatisierten (mmunpräzipitatreaktion')
}

\author{
Von W. Prellwitz, S. Kapp und D. Müller

\section{Zentrallaboratorium der Medizinischen Kliniken und Institut für Medisinische Statistik und Dokumentation! der Universität Mainz}

(Eingegangen am 8. Mai/8. Juli 1974)

\begin{abstract}
Dic Bestimmung der Immunglobuline G, A, M, des Haptoglobins und Transferrins Wurde mit Hilfe der automatisierten Imımunpräzipitatreaktion nephelometrisch mit dem Autoanalyser II und dem Nephelonuorometer durchgeführt. Dabei wurden vier versehiedene Methoden mitcinander verglichen:
\end{abstract}

Methode $A=$ Vorverdūnnung der Proben und Antiseren mit $0.15 \mathrm{~mol} / 1 \mathrm{NaCl}$

Methode $B=$ Vorverdünnung der Proben mit $0,15 \mathrm{~mol} / 1 \mathrm{NaCl}$

Vorverdünnung der Antiseren mit $0,15 \mathrm{~mol} / \mathrm{l} \mathrm{NaCl}+40 \mathrm{~g} / \mathrm{l}$ Polyäthylenglycol 6000

Methode $C=$ Vorverdünnung der Proben und Antiseren mit Britron-Robinson-NaCl-Puffer $75.4 \mathrm{mmol} / 1 \mathrm{pH} 6.0$

Methode $D=$ Vorverdünnung der Proben mit Brition-Robinson- $\mathrm{NaCl}-$ Puffer $75.4 \mathrm{mmol} / 1 \mathrm{pH} 6.0$

Vorverdünnung der Antiseren mit Britron-Robinson-NaCl-Puffer 75,4 mmol/1 pH $6.0+40$ g/l Poly:äthylengly:col 6000

Dic Korrelationen zwischen den verschiedenen Methoden sind hoch signifikant. Durch Zugabe von Polyäthylenglyeol in 0.15 mol/1 $\mathrm{NaCl}$ konnten in Bezug auf Empf indlichkeit, Peak-Höhen, Inkubationszeiten und Einsparung von Antiseren die günstigsten Erǧebnissi erzielt werden. Dic Analysen erfolgten unter statistischer Qualitätskontrolle.

Es ergaben sich folgende Normbereiche bei 292 Männern und 261 Frauen ( 96 Perzentilgrenzen):

IgG: 8-18 g/l (Männer), 7-17 g/l (Frauen),

lgA: $0,75-3,7 \mathrm{~g} / 1$ (Männer), $0,75-3,25 \mathrm{~g} / \mathrm{l}$ (Frauen),

IgM: 0,35-2,0 g/l (Männer), 0,46-2,16 g/l (Frauen),

Haptoglobin: $0,5-2,25 \mathrm{~g} / 1$ (Männer), 0,45-2,07 g/l (Frauen),

Transferrin: $2,45-4,25 \mathrm{~g} / 1$ (Männer), 2,35-4,20 g/l (Fraucn).

Comparative methods for the quantitative immunological determination and normal values of the Immunoglobulins $G, A, M$, Haptoglobin and Transferrin with the automated Immuno-Precipitin-Reaction

The determination of the Immunoglobulins G, A, M, Haptoglobin and Transferrin was carricd out using the automated ImmunoPrecipitin-Reaction with an Autoanalyser II equipped with a fluoronephelometer. The following methods were compared:

Method $A=$ Antigen and antibody dilutions made with $0.15 \mathrm{~mol} / 1 \mathrm{NaCl}$

Method $\mathrm{B}=$ Antigen dilutions made with $0.15 \mathrm{~mol} / 1 \mathrm{NaCl}$

Antibody dilutions made with $0.15 \mathrm{~mol} / 1 \mathrm{NaCl}$ containing $40 \mathrm{~g} / 1$ Poly aethylenglycol 6000

Method C = Antigen and antibody dilutions made with Britton-Robinson-NaCl-buffer $75.4 \mathrm{mmol} / 1 \mathrm{pH} 6.0$

Method D = Antigen dilutions made with Britton-Robinson-NaCl-buffer $75.4 \mathrm{mmol} / 1 \mathrm{pH} 6.0$

antibody dilutions made with Britton-Robinson-NaCl-buffer $75.4 \mathrm{mmol} / 1 \mathrm{pH} 6.0$ containing $40 \mathrm{~g} / 1$ Polyacthylenglycol

The correlations between the various methods is statisticly significant. The results show, that method B with polyaethylenglycol is superior in respect of sensitivity, limit of detection, peak height, saving of antisera and reduction of incubation time in the system. Statistical quality control was applied to the analyses. The following normal values in 292 men and 261 women were obtained (96-percentil-limit):

IgG: $8-18 \mathrm{~g} / \mathrm{l}(\mathrm{men}), 7-17 \mathrm{~g} / 1$ (women),

IgA: $0.75-3.7 \mathrm{~g} / \mathrm{l}$ (men), $0.75-3.25 \mathrm{~g} / 1$ (women),

IgM: $0.35-2.0 \mathrm{~g} / 1$ (men), $0.46-2.16 \mathrm{~g} / 1$ (women),

Haptoglobin: $0.5-2.25 \mathrm{~g} / 1$ (men), $0.45-2.07 \mathrm{~g} / 1$ (women),

Transferrin: $2.45-4.25 \mathrm{~g} / \mathrm{l}$ (men), $2.35-4.20 \mathrm{~g} / 1$ (women).

Die Beurteilung einzelner Serumproteine gewinnt für die Diagnose, Differentialdiagnose und Verlaufskontrolle verschiedener Erkrankungen zunehmend an Bedeutung. Für die quantitative Bestimmung von Serumproteinen stehen heute im allgemeinen folgende Methoden zur Verfügung: radiale Immunodiffusion, Raketenimmunelektrophorese und chemische Bestimmungen. Die Nach- teile dieser Methoden sind zum Teil erwiesen. Die Bestimmungen sind zeitaufwendig, teuer und häufig unspezifisch. Von zahlreichen Untersuchern (1-8) wurde die Bestimmung der bei der Antigen-Antikörperreaktion

1.) Mit freundlicher Unterstützung der Deutschen liorschungsgemeinschaft im Rahmen dos SFB 36. 
entstehenden Immunpräzipitate mit Hilfe der Nephelometrie beschrieben. Durch die Einführung des Autoanalysers II und des Fluoronephelometers konnten die Messungen der Immunpräzipitatreaktion mechanisiert werden. Diese Methode wird heute allgemein als automatisierte Immunpräzipitatreaktion bezeichnet.

In dieser Arbeit wurden mit dem Autoanalyser II zur Bestimmung der Immunglobuline, des Haptoglobins und Transferrins vier verschiedene Methoden miteinander verglichen.

\section{Methodik}

Geräte

Autoanalyser II der Firma Technicon Instruments Corporation Tarrytown, N. Y. USA bestehend aus Probenpumpe (Sampler IV), Proportioning Pump III, Manifold, Transformer, Fluoronephelometer II mit Durchflußzelle 013-B0008-01 und Schreiber.

\section{Reagenzien}

\section{Referenzseren}

A. I. P. Reference Serum (Human) T 03-0495-51 der Firma Technicon

\section{Antiseren}

Anti-Human-Sera von der Ziege der Firma Technicon. StandardHuman-Serum stabilisiert ORDT Behring Werke zur Richtigkeitsund Qualitätskontrolle.

Polyäthylenglycol 6000 Art. 807491 Merck-Schuchardt Britton-Robinson-NaCl-Puffer 75,4 mmol/1 pH 6,0

Lösung 1 :

2,85 mmol/1 Citronensäure nach Sörensen (Merck)

$2,85 \mathrm{mmol} / 1$ Monokaliumhydrogenphosphat p. a. (Merck)

2,85 mmol/1 Barbital p. a. (Merck)

$2,85 \mathrm{mmol} / 1$ Borsäure nach Sörensen (Merck)

$61,6 \mathrm{mmol} / 1 \mathrm{NaCl}$

Lösung 2:

$20 \mathrm{mmol} / 1 \mathrm{Natriumhydroxid-Plätzchen} \mathrm{p.} \mathrm{a.} \mathrm{(Merck)}$

$61,6 \mathrm{mmol} / 1 \mathrm{NaCl}$

Lösung 1 wird mit Lösung 2 auf $\mathrm{pH}$ 6,0 eingestellt.

Vorgehen

Jede Messung wurde mit einer manuellen Vorverdünnung der Serumproben im Verhältnis 1:100 und 1:300 vorgenommen. Die Proben wurden sämtlich durch ein Milliporefilter GSW 02500 $0,22 \mu \mathrm{m}$ filtriert. Dabei wurden folgende Verdünnungslösungen benutzt:

Methode A: Vorverdünnung von Proben und Antiseren mit $0,15 \mathrm{~mol} / 1 \mathrm{NaCl}$

Methode B: Vorverdünnung der Proben mit $0,15 \mathrm{~mol} / 1 \mathrm{NaCl}$ Vorverdünnung des Antiserum mit $0,15 \mathrm{~mol} / 1$ $\mathrm{NaCl}+40 \mathrm{~g} / 1$ Polyäthylenglycol 6000

Methode C: Vorverdünnung von Proben und Antiseren mit Britton-Robinson-NaCl-Puffer 75,4 mmol pH 6,0

Methode D: Vorverdünnung der Proben mit Britton-Robinson$\mathrm{NaCl}$-Puffer 75,4 mmol/1 pH 6,0

Vorverdünnung der Antiseren mit Britton-Robin son- $\mathrm{NaCl}$-Puffer $75,4 \mathrm{mmol} / 1 \mathrm{pH} 6,0+40 \mathrm{~g} / \mathrm{l}$ Polyäthylenglycol 6000 .

Allen Verdünnungslösungen wurde $1 \mathrm{ml} / 1$ Tween 20 zugesetzt.

Das Fließschema und die Durchflußgeschwindigkeiten sind in Abbildung 1 dargestellt. Bei Verwendung von Polyäthylenglycol betrug die Inkubationszeit $4 \mathrm{~min}$, ohne Polyäthylenglycol 17 min. Die Verdünnungen der entsprechenden Antisera für die einzelnen Methoden sind in Tabelle 1 aufgeführt. mit Polyäthylenglykol

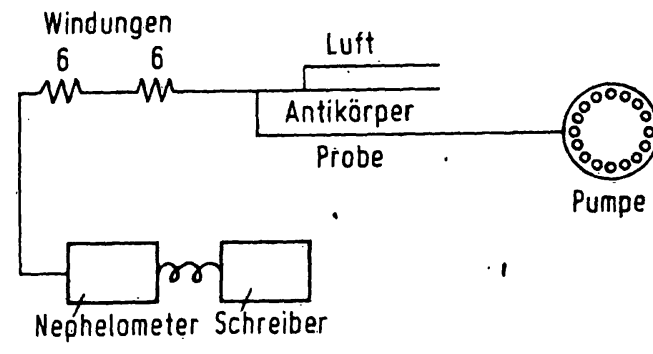

ohne Polyäthylenglykol

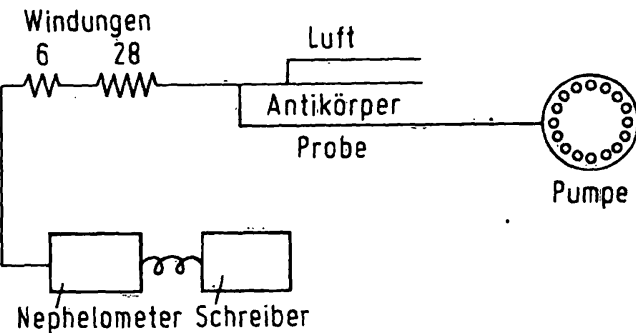

Abb. 1. Fließschema und Durchflußvolumina zur quantitativ immunologischen Bestimmung von Serumproteinen mit Hilfe der automatisierten Immunpräzipitatreaktion mit und ohne Polyäthylenglycol 6000.

Durchflußgeschwindigkeiten in $\mathrm{ml} / \mathrm{min}$ für die angegebenen Methoden

\begin{tabular}{|c|c|c|c|c|c|}
\hline & IgG & IgA & IgM & $\begin{array}{l}\text { Hapto- } \\
\text { globin }\end{array}$ & $\begin{array}{l}\text { Trans- } \\
\text { ferrin }\end{array}$ \\
\hline robe & 0,03 & 0,01 & 0,01 & 0,03 & 0,03 \\
\hline ntiserum & 0,32 & 0,32 & 0,32 & 0,32 & 0,32 \\
\hline uft & 0,32 & 0,32 & 0,32 & 0,32 & 0,32 \\
\hline Pumpe & 0,02 & 0,02 & 0,02 & 0,02 & $0 ; 02$ \\
\hline
\end{tabular}

Tab. 1. Verdünnungen der Antiseren zur Bestimmung der Immunglobuline, des Haptoglobins und Transferrins bei vier verschiedenen Methoden der automatisierten Im. munpräzipitatreaktion.

\begin{tabular}{lllll}
\hline Methode & A & B & C & D \\
\hline IgG & $1: 40$ & $1: 100$ & $1: 40$ & $1: 100$ \\
IgA & $1: 40$ & $1: 40$ & $1: 40$ & $1: 40$ \\
IgM & $1: 25$ & $1: 40$ & $1: 25$ & $1: 40$ \\
Haptoglobin & $1: 40$ & $1: 100$ & & \\
Transferrin & $1: 40$ & $1: 110$ & & \\
\hline
\end{tabular}

Für die Ermittlung der Normbereiche wurden bei insgesamt 292 Männern und 261 Frauen die fünf verschiedenen. Serumproteine bestimmt. Diese Probanden wurden poliklinisch voruntersucht, außerdem bestimmten wir parallel noch weitere klinisch-chemische, hämatologische und gerinnungsphysiologische Parameter, um jede Erkrankung ausschließen zu können.

\section{Ergebnisse}

Die Peak-Höhen in mim in Abhängigkeit von der Antigenkonzentration und den verschiedenen Methoden sind in Abbildung 2 ersichtlich. Sowohl bei einer Serumverdünnung von $1: 100$ und $1: 300$ sind bis zu einer bestimmten Antigenkonzentration die Peak-Höhen bei Methode $\mathrm{B}(0,15 \mathrm{~mol} / \mathrm{l} \mathrm{NaCl}+40 \mathrm{~g} / \mathrm{l}$ Polyäthylenglycol $)$ am 

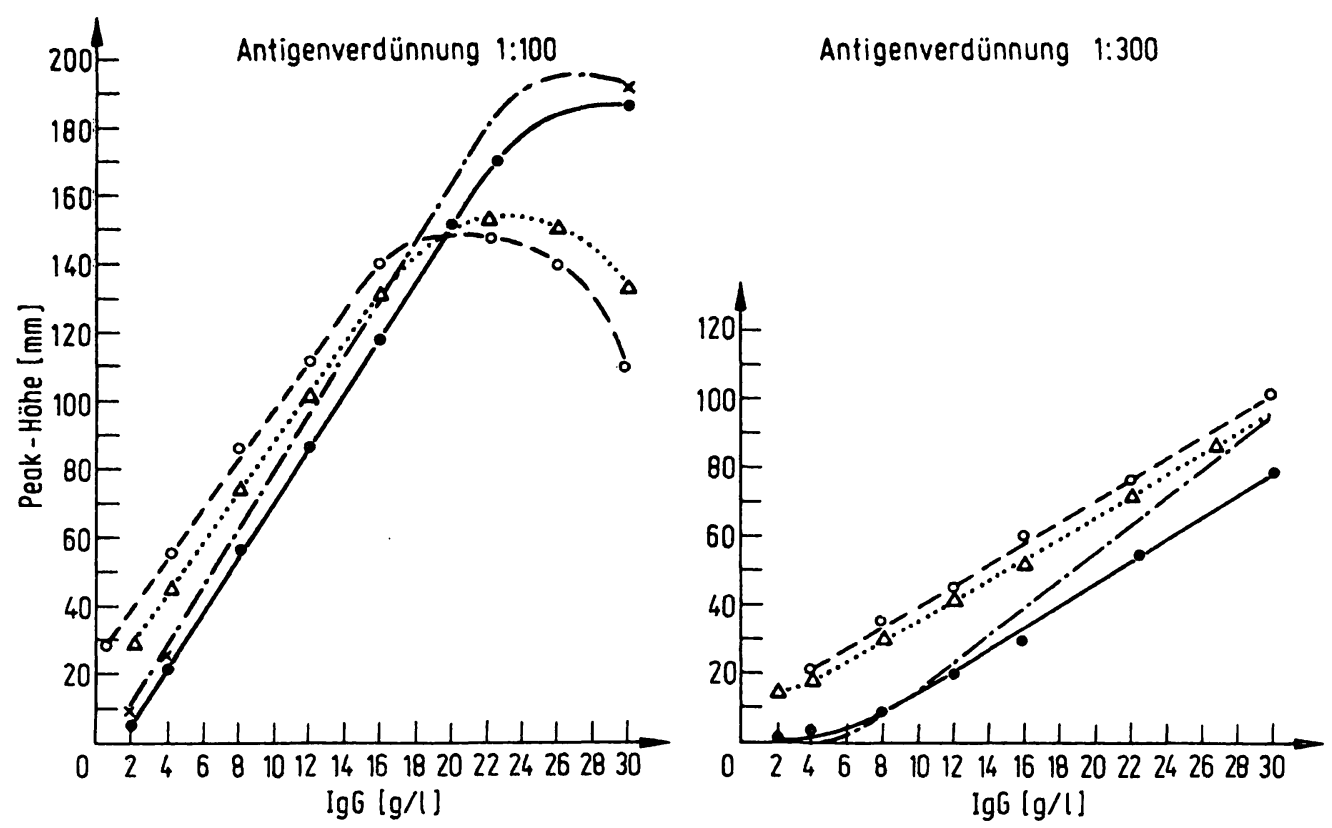

Abb. 2. Peakhöhen in $\mathrm{mm}$ bei vier verschiedenen Methoden der IgG-Bestimmung mit Hilfe der automatisierten Immunpräzipitatreaktion. -— $0,15 \mathrm{~mol} / 1 \mathrm{NaCl}$ (A)

○- $0 \quad 0,15 \mathrm{~mol} / 1 \mathrm{NaCl}+40 \mathrm{~g} / 1$ Polyäthylenglycol (B)

-.-.- 75,4 mmol/1 Britton-Robinson-NaCl-Puffer pH 6,0 (C)

$\Delta \cdots . . . \Delta \quad 75,4 \mathrm{mmol} / 1$ Britton-Robinson-NaCl-Puffer pH 6,0 $+40 \mathrm{~g} / 1$ Polyäthylenglycol (D)

höchsten. Damit ist die Empfindlichkeit dieser Methode am größten. Ähnliche Verhältnisse liegen auch bei der Bestimmung der Immunglobuline A, M, des Haptoglobins und Transferrins vor.

In Tabelle 2 sind die oberen und unteren Nachweisgrenzen der Immunglobuline mit der Methode B aufgeführt. Bei der routinemäßigen Bestimmung der Immunglobuline mit einer manuellen Vorverdünnung der Proben von $1: 100$ und 1:300 können alle in der Klinik wichtigen quantitativen Serumprotẹinveränderungen erfaßt werden.

Die Tabelle 3 zeigt die Ergebnisse der Korrelationsrechnungen der Serumproteinbestimmungen mit Hilfe verschiedener Methoden der automatisierten Immunpräzipitatreaktion. Dạbei ergẹben sich zwischen den Methoden keine Unterschiede. Ähnliche Ergebnisse wurden von anderen Untersuchern publiziert $(3,4,8,9)$.

Die Präzision in der Serie wurde mit dem Standard Human-Serum der Behringwerke überprüft. Bei der Verwendung von Polyäthylenglycol in $0,15 \mathrm{~mol} / 1 \mathrm{NaCl}$ (Methode B) für die Bestimmungen der fünf Serumproteine lagen die Variationskoeffizienten zwischen 2,5-4,1 (Tab. 4).

Die Präzision von Tag zu Tag wurde bisher funf Monate lang durchgeführt. Die Variationskoeffizienten liegen hier selbstverständlich höher als bei der Präzision in der Serie. In Tabelle 5 sind die entsprechenden Werte für die fünf Serumproteine aufgezeichnet.

Die Normbereiche der untersuchten Proteine bei 292 gesunden Männern und 261 Frauen sind in Tabelle 6 dargelegt. Die Verteilung der Werte ist log
Tab. 2. Untere (I) und obere (II) Nachweisgrenzen der Immunglobuline im Serum in $\mathrm{g} / \mathrm{l}$. Methode $\mathrm{B}(0,15 \mathrm{~mol} / 1 \mathrm{NaCl}+40 \mathrm{~g} / 1$ Polyäthylenglycol)

\begin{tabular}{lllllll}
\hline & \multicolumn{1}{c}{ IgG } & \multicolumn{1}{c}{ IgM } & \multicolumn{1}{c}{ IgA } \\
\hline Probenverdünnung & $1: 100$ & $1: 300$ & $1: 100$ & $1: 300$ & $1: 100$ & $1: 300$ \\
I & 1,0 & 4,0 & 0,2 & 0,4 & 0,2 & 0,5 \\
II & 16 & 44 & 2,4 & 7,0 & 3,5 & 9,0 \\
\hline
\end{tabular}

Tab. 3. Korrelationskoeffizienten $\mathrm{r}$ zwischen verschiedenen Serumprotcinbestimmungen (Methode $A, B, C, D$ ) mit Hilfe der automatisierten Immunpräzipitatreaktion.

\begin{tabular}{lllll}
\hline Protein & Methoden & $\mathrm{n}$ & $\mathrm{r}$ & Signifikanz \\
\hline \multirow{4}{*}{ IgG } & A/B & 150 & 0,895 & ++ \\
& A/C & 144 & 0,950 & ++ \\
& A/D & 144 & 0,871 & ++ \\
& & & & \\
IgM & A/B & 110 & 0,855 & + \\
& A/C & 80 & 0,912 & ++ \\
& A/D & 80 & 0,865 & ++ \\
& & & & \\
IgA & A/B & 140 & 0,932 & ++ \\
& A/C & 140 & 0,867 & ++ \\
& A/D & 140 & 0,913 & ++ \\
\hline
\end{tabular}

normal. Zwischen Männern und Frauen bestehen keine signifikanten Unterschiede. Werden die Ergebnisse in den verschiedenen Altersgruppen bei Frauen und Männern miteinander verglichen, so zeigt sich bei den Immunglobulinen IgG und IgM ein Trend: mit zunehmendem Alter steigen die arithmetischen Mittelwerte in diesen Gruppen an. 
Tab. 4. Präzision in der Seric bei der immunologischen Bestimmung von Serumproteinen (Standard-Human-Serum, Behring Werke

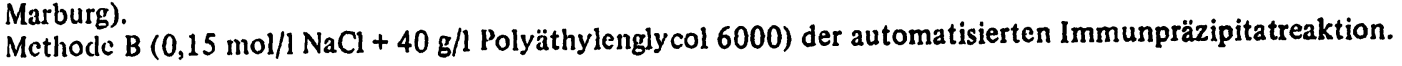

\begin{tabular}{|c|c|c|c|c|c|c|c|c|c|c|c|c|c|c|c|c|c|c|c|}
\hline \multirow{3}{*}{ Sollwert $[g / 1] \bar{X} \pm S$} & \multicolumn{3}{|c|}{ IgG } & \multicolumn{4}{|c|}{$\operatorname{Ig} A$} & \multicolumn{4}{|c|}{ IgM } & \multicolumn{4}{|c|}{ Haptoglobin } & \multicolumn{4}{|c|}{ Transferrin } \\
\hline & \multicolumn{3}{|c|}{$11,0 \pm 0,82$} & \multicolumn{4}{|c|}{$2,4 \pm 0,18$} & \multicolumn{4}{|c|}{$1,8 \pm 0,06$} & \multicolumn{4}{|c|}{$1,88 \pm 0,08$} & \multicolumn{4}{|c|}{$3,4 \pm 0,14$} \\
\hline & $\begin{array}{l}\bar{x} \\
{[\mathrm{~g} / \mathrm{l}]}\end{array}$ & $\begin{array}{l}S \\
{[\mathrm{~g} / \mathrm{l}]}\end{array}$ & $\begin{array}{l}\text { VK } \\
{[\%]}\end{array}$ & $\mathrm{N}$ & $\begin{array}{l}\bar{x} \\
{[\mathrm{~g} / \mathrm{l}]}\end{array}$ & $\begin{array}{l}S \\
{[\mathrm{~g} / \mathrm{I}]}\end{array}$ & $\begin{array}{l}\text { VK } \\
{[\%]}\end{array}$ & $\mathrm{N}$ & $\begin{array}{l}\bar{X} \\
{[\mathrm{~g} / \mathrm{l}]}\end{array}$ & $\begin{array}{l}S \\
{[g / 1]}\end{array}$ & $\begin{array}{l}\text { VK } \\
{[\%]}\end{array}$ & $\mathbf{N}$ & $\begin{array}{l}\bar{X} \\
{[\mathrm{~g} / \mathrm{l}]}\end{array}$ & $\begin{array}{l}S \\
{[\mathrm{~g} / \mathrm{l}]}\end{array}$ & VK & $\mathbf{N}$ & $\begin{array}{l}\bar{X} \\
{[\mathrm{~g} / \mathrm{l}]}\end{array}$ & $\begin{array}{l}S \\
{[\mathbf{g} / \mathbf{l}] .}\end{array}$ & $\begin{array}{l}\text { VK } \\
{[\%]}\end{array}$ \\
\hline
\end{tabular}

Serum-

verdünnungen

$1: 100$

$\begin{array}{lllllllllllllllllllll}100 & 11,1 & 0,275 & 2,5 & 80 & 2,24 & 0,067 & 2,8 & 60 & 1,81 & 0,051 & 2,83 & 75 & 1,83 & 0,06 & 3,3 & 90 & 3,42 & 0,085 & 2,5\end{array}$

$1: 300$

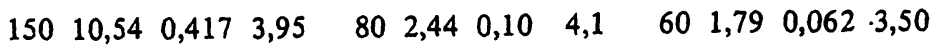

Tab. 5. Präzision von Tag zu Tag innerhalb von 5 Monaten (1-5) bei der immunologischen Bestimmung von Serumprotcinen (Standard-Human-Scrum, Behring Werke Marburg).

Methode $\mathrm{B}(0,15 \mathrm{~mol} / \mathrm{l} \mathrm{NaCl}+40 \mathrm{~g} / 1$ Polyäthylenglycol 6000) der automatisicrten Immunpräzipitatreaktion.

\begin{tabular}{|c|c|c|c|c|c|c|c|c|c|c|c|c|c|c|c|c|c|c|c|c|}
\hline \multirow[t]{2}{*}{$\begin{array}{l}\text { Sollwert } \\
{[\mathrm{g} / 1]} \\
\mathrm{X} \pm \mathrm{S}\end{array}$} & \multicolumn{3}{|c|}{$\begin{array}{c}\operatorname{Ig} G \\
12,8 \pm 0,51\end{array}$} & \multirow[b]{2}{*}{$\begin{array}{l}\text { VK } \\
{[\%]}\end{array}$} & \multicolumn{3}{|c|}{$\begin{array}{c}\operatorname{IgA} \\
2,3 \pm 0,09\end{array}$} & \multicolumn{4}{|c|}{$\begin{array}{c}\operatorname{IgM} \\
0,94 \pm 0,04\end{array}$} & \multirow[b]{2}{*}{$\begin{array}{l}\text { VK } \\
{[\%]}\end{array}$} & \multicolumn{4}{|c|}{$\begin{array}{l}\text { Haptoglobin } \\
1,88 \pm 0,08\end{array}$} & \multicolumn{4}{|c|}{$\begin{array}{l}\text { Transferrin } \\
3,4 \pm 0,14\end{array}$} \\
\hline & $\mathrm{N}$ & $\begin{array}{l}\bar{X}: \\
{[\mathrm{g} / 1]}\end{array}$ & $\begin{array}{l}S \\
{[g / l]}\end{array}$ & & $\mathrm{N}$ & $\begin{array}{l}\bar{X} \\
{[g / 1]}\end{array}$ & $\begin{array}{l}S \\
{[\mathrm{~g} / \mathrm{l}]}\end{array}$ & $\begin{array}{l}\text { VK } \\
{[\%]}\end{array}$ & $\mathrm{N}$ & $\begin{array}{l}\bar{X} \\
{[g / 1]}\end{array}$ & $\begin{array}{l}S \\
{[g / 1]}\end{array}$ & & $N$ & $\begin{array}{l}\bar{X} \\
{[\mathrm{~g} / \mathrm{l}]}\end{array}$ & $\begin{array}{l}S \\
{[g / 1]}\end{array}$ & $\begin{array}{l}\text { VK } \\
{[\%]}\end{array}$ & $\mathrm{N}$ & $\begin{array}{l}\bar{X} \\
{[\mathrm{~g} / \mathrm{l}]}\end{array}$ & $\begin{array}{l}S \\
{[g / 1]}\end{array}$ & $\begin{array}{l}\text { VK } \\
{[\%]}\end{array}$ \\
\hline 1 & 36 & 12,5 & 0,56 & 4,5 & 36 & 2,29 & 0,11 & 5,0 & 36 & 0,96 & 0,06 & 6,03 & 28 & 1,92 & 0,08 & 4,6 & 28 & 3,39 & 0,14 & 4,3 \\
\hline 2 & 28 & 12,6 & 0,45 & 3,6 & 28 & 2,33 & 0,11 & 4,8 & 28 & 0,90 & 0,07 & 6,59 & 20 & 1,90 & 0,12 & 6,5 & 26 & 3,43 & 0,14 & 4,2 \\
\hline 3 & 38 & 12,7 & 0,62 & 4,9 & 38 & 2,31 & 0,11 & 4,7 & 36 & 0,98 & 0,05 & 5,53 & 30 & 1,85 & 0,12 & 6,5 & 33 & 3,31 & 0,16 & 4,8 \\
\hline 4 & 26 & 12,5 & 0,59 & 4,8 & 36 & 2,31 & 0,15 & 6,6 & 28 & 0,95 & 0,06 & 7,06 & 25 & 1,79 & 0,11 & 6,1 & 30 & 3,46 & 0,15 & 4,3 \\
\hline 5 & 32 & 12,5 & 0,38 & 3,1 & 32 & 2,33 & 0,11 & 4,8 & 30 & 0,98 & 0,06 & 6,43 & 28 & 1,90 & 0,09 & 5,1 & 32 & 3,49 & 0,14 & 4,2 \\
\hline
\end{tabular}

Tab. 6. Normberciche der Serumkonzentrationen der Immunglobuline IgG, IgA, IgM, des Haptoglobins und Transferrins bei gesunden Männern und Frauen.

\begin{tabular}{|c|c|c|c|c|c|c|c|c|c|c|}
\hline & \multicolumn{2}{|c|}{ IgG } & \multicolumn{2}{|c|}{ IgA } & \multicolumn{2}{|c|}{$\operatorname{IgM}$} & \multicolumn{2}{|c|}{ Haptoglobin } & \multicolumn{2}{|c|}{ Haptoglobin } \\
\hline & Männer & Frauen & Münner & Frauen & Männer & Frauen & Männer & Frauen & Männer & Frauęn \\
\hline $\mathrm{N}$ & 292 & 261 & 292 & 261 & 292 & 261 & 292 & 261 & 292 & 261 \\
\hline $\bar{X}[g / 1]$ & 12,51 & 11,52 & 2,12 & 1,82 & 1,09 & 1,21 & 1,32 & 1,21 & 3,30 & 3,27 \\
\hline$S[g / 1]$ & 2,9 & 2,8 & 0,81 & 0,76 & 0,49 & 0,50 & 0,50 & 0,49 & 0,49 & 0,49 \\
\hline $\begin{array}{l}96 \\
\text { Perzentil- } \\
\text { grenzen } \\
{[\mathrm{g} / \mathrm{l}]}\end{array}$ & $8,0-18,0$ & $7,0-17,0$ & $0,75-3,7$ & $0,75-3,25$ & $0,35-2,0$ & $0,46-2,16$ & $0,5-2,25$ & $0,45-2,07$ & $2,45-4,25$ & $2,35-4,20$ \\
\hline
\end{tabular}

\section{Diskussion}

Für die quantitative nephelometrische Bestimmung immunologischer Antigen-Antikörper Reaktionen müssen optimale Reaktionsbedingungen gefunden werden, um hohe Streulichtintensitäten zu erhalten. Nach Untersuchungen von Ebeling (9) sind für die Nephelometrie besonders die Ionenstärke und der pH-Wert der Verdünnungslösungen sowie die Inkubationszeiten wichtig. Bei der Bestimmung des Transferrin konnten diese Bedingungen nur mit einem 75,4 mmol/1 Puffer pH 6,0 erreicht werden. Ritchie (10) dagegen beschrieb als günstigste Verdünnungslösung für Proben und Antiseren $0,15 \mathrm{~mol} / 1 \mathrm{NaCl}$. In den hier aufgefuihrten 
Untersuchungen konnten zwischen der $0,15 \mathrm{~mol} / 1$ $\mathrm{NaCl}$ und dem 75,4 mmol/1 Puffer pH 6,0 in Bezug auf Korrelationskoeffizienten, Richtigkeit und Präzision in der Serie und von Tag zu Tag keine Unterschiede gefunden werden. Die Peakhöhen differieren nur geringfügig zwischen beiden Methoden.

Von erheblicher Bedeutung für die Immunpräzipitatreaktion ist dagegen die Verwendung von Polyäthylenglycol. Nach bisher vorliegenden Untersuchungen (11-14) vermutet man einen Einfluß auf die Präzipitation löslicher Antigen-Antikörper-Komplexe und eventuell eine Konstanterhaltung der Präzipitatteilchen. Ob dabei Änderungen der sterischen Konfiguration der Antigen-Antikörper-Komplexe eine Rolle spielen, wird vermutet.

Wir haben, anlehnend an die Methoden A und B, in $0,15 \mathrm{~mol} / 1 \mathrm{NaCl}$ mit und ohne Verwendung von Polyäthylenglycol bei konstanten Antikörperkonzentrationen eine Heidelberger-Kurve (15) gemessen. Dabei konnte eine Verschiebung des Äquivalenzpunktes zur geringeren Antigenkonzentration durch Polyäthylenglycol beobachtet werden (Abb. 3). Dieser Befund spräche für eine

\section{Literatur}

1. Ginthin, D. \& Edelloch, H. (1951), J. Immunol. 66, 57-77.

2. Larson, C., Orenstein, P. \& Ritchie, R. F. (1970), Thurman Assoc. Miami, Florida 9-17.

3. Larson, C., Orenstein, P. \& Ritchic, R. F. (1971), Adv. Aut. Anal. 1, 101-107.

4. Larson, C., Gorman, J. \& Becker, A. (1972), Techn. Intern. Cong. N. Y. 21-23.

5. Riccomi, H., Masson, P. \& Heremans, J. F. (1972), Techn. Intern. Cong. 53, N. Y. 5-9.

6. Ritchie, R. F. (1967), J. Lab. Clin. Med. 70, 512-519.

7. Marcroft, J. \& Newland, L. M. (1973), Clin. Chim. Acta 46, 399-405.
Präzipitation löslicher Antigen-Antikörper-Komplexe und könnte den geringeren Verbrauch an Antiseren und die kürzere Inkubationszeiten erklären.

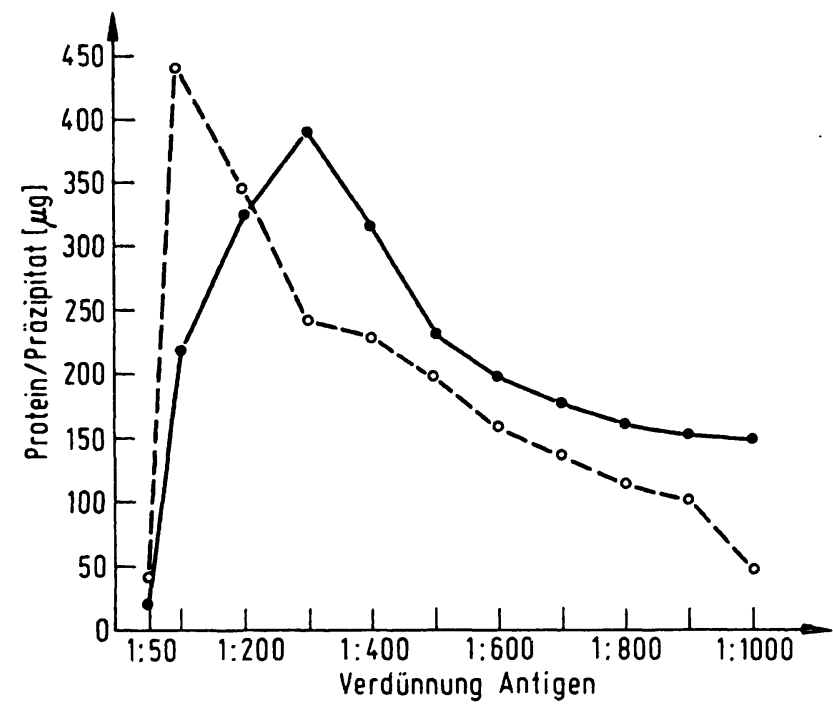

Abb. 3. Heidelberger-Kurve: IgG-Globuline $\mu \mathrm{g}$ Protein im Präzipitat bei konstanter Antikörperkon-

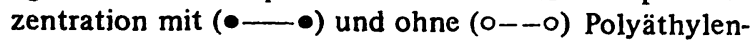
glycol.
8. Killingsworth, L. M. \& Savory, J. (1971), Clin. Chem. 17, 936-940.

9. Ebeling, H. (1973), diese Z. 11, 209-214.

10. Ritchie, R. F., Alper, C. A., Graves, J., Pearson, N. \& Larson, C. (1973), Amer. J. Clin. Path. 59, 151-157.

11. Hellsing, K. (1969), Biochem. J. 112, 475-481.

12. Hellsing, K. (1969), Biochem. J. 112, 483-487.

13. Hellsing, K. (1972), Techn. Intern. Cong., 17-20 (N. Y.).

14. Creighton, W. D., Lambert, P. H. \& Mischer, P. A. (1973), J. Immunol. 111, 1219-1227.

15. Heidelberger, M. \& Kendall, F. E. (1935), J. Exp. Med. 62, 167-169. 